\title{
Serum human epididymis protein 4 levels in colorectal cancer patients
}

\author{
YASEMIN KEMAL ${ }^{1}$, GUZIN DEMIRAG $^{2}$, ABDULKERIM BEDIR $^{3}$, LEMAN TOMAK $^{4}$, \\ MURAT DEREBEY ${ }^{5}$, DILEK ERDEM ${ }^{6}$, UFUK GÖR $^{7}$ and IDRIS YÜCEL ${ }^{8}$ \\ ${ }^{1}$ Department of Medical Oncology, Samsun Training and Research Hospital, Samsun 55100; Departments of ${ }^{2}$ Medical Oncology, \\ ${ }^{3}$ Biochemistry, ${ }^{4}$ Biostatistics and ${ }^{5}$ General Surgery, Faculty of Medicine, Ondokuz Mayis University, Samsun 55270; \\ ${ }^{6}$ Department of Medical Oncology, Medical Park Samsun Hospital, Samsun 55200; Departments of ${ }^{7}$ Biochemistry \\ and ${ }^{8}$ Medical Oncology, Faculty Of Medicine, Ondokuz Mayis University, Samsun 55270, Turkey
}

Received October 25, 2016; Accepted July 4, 2017

DOI: $10.3892 / \mathrm{mco} .2017 .1332$

\begin{abstract}
Tumour markers are widely used for the diagnosis, staging and monitoring of colorectal cancer (CRC) patients in clinical practice. Carcinoembryonic antigen (CEA) and carbohydrate antigen 19-9 (CA19-9) are the most frequently used biomarkers in CRC patients. A number of studies have recently investigated the presence of human epididymis protein 4 (HE4) overexpression in certain cancer types. Its significance in ovarian and endometrial cancer has been well-demonstrated. The aim of the present study was to evaluate the significance of serum HE4 levels in CRC patients. A total of 46 newly diagnosed CRC patients and 36 age- and gender-matched healthy subjects were included in the study. The concentrations of CEA and CA19-9 were also determined and compared according to HE4 levels. HE4 positivity was determined in 13 of the 46 cases $(28.3 \%)$ in the CRC group, but no HE4-positive subjects were identified in the control group $(0 \% ; \mathrm{P}=0.009)$. The area under the receiver operating characteristic curve for HE4 positivity was 0.641 (95\% CI: $0.523-0.760)$. HE4 was statistically significantly positive in patients with stage III-IV disease and in those with high CA 19-9 levels (all $\mathrm{P}<0.01$ ). To the best of our knowledge, this is the first study to investigate HE4 expression in CRC patients, and the findings suggest that it may be a useful biomarker, particularly in stage III-IV patients.
\end{abstract}

\section{Introduction}

Colorectal cancer (CRC) is the third most common cancer in men and the second in women worldwide (1). According

Correspondence to: Dr Yasemin Kemal, Department of Medical Oncology, Samsun Training and Research Hospital, 199 Baris Street, Samsun 55100, Turkey

E-mail: drturkmen@yahoo.com

Key words: colorectal cancer, human epididymis protein 4, tumour marker to the World Health Organization GLOBOCAN database, in 2012, 1.4 million new cases of CRC were diagnosed and 694,000 patients succumbed to this disease (2). CRC is one of the major causes of cancer-related mortality, with a 5 -year survival of $~ 50 \%$ (3). Metastasis to the liver and/or lungs is the main cause of death and is already present in $\leq 25 \%$ of patients at presentation (4). Early diagnosis and timely intervention are crucial for therapeutic effectiveness and prolongation of survival. Adequate preoperative staging is important for the management of CRC patients so that the appropriate treatment plan may be devised. Following treatment completion, patients must be monitored for the development of recurrence or distant metastasis. Tumour markers are widely used for the diagnosis, staging and monitoring of CRC patients in clinical practice. These markers are biological or biochemical substances that are usually produced by tumour cells and then secreted into the circulation in detectable amounts and used for screening and staging (5). Carcinoembryonic antigen (CEA), an oncofetal glycoprotein that is overexpressed in adenocarcinoma of the colorectum, is the most frequently used biomarker in CRC (6). CEA has low sensitivity and specificity for diagnosis; therefore, it should not be used for screening. However, in patients with newly diagnosed CRC, the absolute level of the serum CEA is correlated with disease burden increase in $75 \%$ of patients with distant metastasis and has prognostic value (7). High levels of CEA may also be observed in association with benign conditions, such as smoking, peptic ulcer, inflammatory bowel disease, pancreatitis, hypothyroidism, biliary obstruction and cirrhosis (8). Another tumour marker, carbohydrate antigen 19-9 (CA19-9), is produced by adenocarcinomas of the pancreas, stomach, gallbladder, colon, ovary and lung. The incidence of high serum CA19-9 levels in CRC ranges from 20 to $40 \%$ (9).

At present, the sensitivity and specificity of the clinically used markers of CRC are low for early diagnosis and screening; thus, a number of studies have been conducted to identify more effective markers. Several studies have recently investigated the overexpression of human epididymis protein 4 (HE4) in certain cancer types. HE4 was first identified in 1991 by Kirckhoff et al (10) and its overexpression has been found in 
carcinomas of the ovary, endometrium, lung, breast, gastrointestinal system and uroepithelium $(11,12)$. HE4 was originally isolated from the epithelium of the distal epididymis and was predicted to be a protease inhibitor essential for sperm maturation $(2,13)$. The HE4 gene product is also referred to as whey acidic protein and four-disulphide core domain protein 2 (14). This protein acts in a manner similar to antiproteinases, secretory leukocyte protease 1 and elafin; they all exhibit antibacterial properties and exert anti-inflammatory effects (15). HE4 is generally considered to play a role in natural immunity; however, its true role in carcinogenesis has not been fully elucidated (10). Multiple studies have reported its specifity and sensitivity as an ovarian cancer biomarker (16). In 2009, the United States Food and Drug Administration (FDA) approved HE4 as a useful marker for monitoring patients with epithelial ovarian cancer (17); and in September, 2011, the FDA also approved marketing of HE4 for the detection of ovarian masses (18). There is accumulating evidence that HE4 may also be a useful marker for endometrial cancer. Recently, HE4 expression was also investigated in breast, lung, gastric and pancreatic cancer (19). To the best of our knowledge, this is the first clinical study to evaluate the diagnostic and preoperative predictive value of serum HE4 levels in newly diagnosed CRC patients.

\section{Patients and methods}

Patients. The study comprised 46 newly diagnosed CRC patients who were diagnosed and treated at the Ondokuz Mayis University Hospital (Samsun, Turkey) between January, 2014 and July, 2014, and 36 age- and gender-matched healthy subjects as the control group. The control subjects were individually selected from patients attending the outpatient clinic for a routine check-up. Approval for the study was granted by the Ethics Committee of the Ondokuz May1s University Hospital and all the patients and controls provided written informed consent.

The pathological staging of the CRC patients was performed according to the 7 th edition of the tumour-node-metastasis (TNM) classification (http://www.uicc.org/sites/main/ files/private/TNM_Classification_of_Malignant_Tumours_ Website_15\%20MAy2011.pdf). Patients with renal failure were excluded, as they have high HE4 levels. All the subjects were treatment-naive (no operation, chemotherapy or chemoradiotherapy) prior to blood sample collection.

Blood sampling and serum marker levels. Blood samples were obtained by venous puncture, centrifuged, and stored at $-50^{\circ} \mathrm{C}$ until the analyses were performed. An ELISA kit (Cusabio Biotech Co., Ltd., Wuhan, China) was used for HE4 evaluation. All measurements were performed according to the manufacturers' instructions. The plates were read at the wavelength of $450 \mathrm{~nm} 10$ min after the administration of the stop solution. The minimum detectable dose of HE4 was $<0.30 \mathrm{pmol} / \mathrm{ml}$ according to this measurement. The HE4 cut-off level of $\leq 0.30 \mathrm{pmol} / \mathrm{ml}$ was considered to be HE4-negative and all cases with HE4 levels $>0.30 \mathrm{pmol} / \mathrm{ml}$ were considered as HE4-positive.

The serum CEA and CA19-9 levels were measured using the enzyme immunoassay method, using a HITACHI automatic analyzer (Hitachi Corporation, Tokyo, Japan). According to the manufacturer's instructions, the normal range for serum CEA was 0-3.4 $\mathrm{ng} / \mathrm{ml}$ and for CA19-9 0-37 U/ml.

Statistical analysis. The statistical analysis was performed with SPSS software, version 17.0 (SPSS Inc., Chicago, IL, USA). The results were expressed as mean \pm standard deviation and frequency (\%). The normality of distribution was checked initially and Student's t-test was then used to compare the mean values. Yates's corrected Chi-squared and Fisher's exact tests were also used for frequency (data obtained by counting) comparisons. Receiver operator characteristics (ROC) curves were applied and the area under the curve (AUC) was calculated to determine the sensitivity and specificity of HE4. $\mathrm{P}<0.05$ was considered to indicate statistically significant differences.

\section{Results}

Subject characteristics. A total of 46 newly diagnosed CRC patients and 36 healthy age- and gender-matched control subjects were included in the study. The demographic characteristics of the patients and controls are shown in Table I. There were no significant demographic differences between the two groups. HE4 positivity was determined in 13 of the 46 cases $(28.3 \%)$ in the CRC group; however, no HE4positive subjects were identified in the control group $(0 \%$; $\mathrm{P}=0.002)$.

Patient characteristics. The detailed clinicopathological characteristics of the CRC patients are shown in Table II. CEA positivity was determined in $28(66.7 \%)$ and CA19-9 positivity in $17(40.5 \%)$ CRC cases. Of the 46 patients, 33 were lymph node-positive and 22 of those had stage IV disease.

Comparison between HE4-positive and-negative patients. As shown in Table III, nodal involvement was significantly associated with HE4 positivity. None of the node-negative patients were HE4-positive, whereas 13 of the 33 patients with nodal involvement were HE4-positive $(\mathrm{P}=0.009)$. When comparing HE4 positivity between cancer stages I-II and III-IV, the difference was statistically significant $(\mathrm{P}=0.009)$. HE4 was also statistically significantly positive in patients with high CA19-9 levels ( $\mathrm{P}<0.001)$. No statistically significant associations were observed between tumour grade, CEA levels and HE4.

Diagnostic performance of HE4. The ROC curve analyses of the diagnostic performance of $\mathrm{HE} 4$ in healthy controls and CRC patients revealed $\mathrm{AUC}=0.641[95 \%$ confidence interval $(\mathrm{CI})$ : $0.523-0.760, \mathrm{P}=0029$; sensitivity $=28.3 \%$, specificity $=100.0 \%$; Fig. 1]. When ROC curve analyses were applied to stage III and IV, the AUC increased to 0.697 (95\% CI): 0.569-0.825, $\mathrm{P}=0.005$; sensitivity $=39.4 \%$, specificity $=100.0 \%$ ). In another ROC curve analysis, HE4 was combined with CEA and CA19-9 and it was demonstrated that the combinations may enhance diagnostic accuracy (AUC of HE4+CEA+CA19-9=0.837, $\mathrm{P}<0.001$; Fig. 2). 
Table I. Characteristics of colorectal cancer patients and controls.

\begin{tabular}{|c|c|c|c|}
\hline Variables & Patients, $\mathrm{n}(\%)(\mathrm{n}=46)$ & Controls, $\mathrm{n}(\%)(\mathrm{n}=36)$ & P-value \\
\hline Age, years $($ mean $\pm S D)$ & $60.5 \pm 11.8$ & $58 \pm 12.5$ & 0.360 \\
\hline Gender & & & 0.901 \\
\hline Female & $16(34.8)$ & $13(36.1)$ & \\
\hline Male & $30(65.2)$ & $23(63.9)$ & \\
\hline HE4 & & & 0.002 \\
\hline Positive & $13(28.3)$ & $0(0.0)$ & \\
\hline Negative & $33(71.7)$ & $36(100.0)$ & \\
\hline
\end{tabular}

Bold print indicates statistical significance. SD, standard deviation; HE4, human epididymis protein 4.

\section{Discussion}

The present study demonstrated that the rate of HE4 positivity in CRC patients is significantly higher compared with that in healthy controls, and this positivity is strongly associated with nodal involvement, advanced stage and CA19-9 positivity.

Although a number of tumour markers are widely used for the diagnosis, staging and monitoring of CRC patients in clinical practice, their role in the early detection of colorectal tumours is limited. Therefore, novel biomarkers with better clinical utility are needed. Serum HE4 was recently introduced in the routine diagnostics of ovarian cancer (20). In September, 2011, the US FDA approved marketing of HE4 for the detection of ovarian masses, as HE4 has a higher sensitivity and specificity compared with CA125 for the diagnosis of ovarian cancer (21).

HE4 was first described by Kirchhoff et al in 1991 (10); it acts as a proteinase inhibitor and belongs to the 'four-disulphide core' family of proteins (10). HE4 is expressed in normal as well as malignant tissues (10). Galgano et al reported that breast epithelium, female genital tract, epididymis, vas deferens, distal renal tubules, respiratory epithelium, colonic mucosa and salivary glands all show HE4 immunoreactivity (12). HE4 expression has also been confirmed in several cancers, including ovarian cancer, mesothelioma, lung, endometrial, breast, gastrointestinal, renal and transitional cell carcinomas (12). The highest expression levels have been found in ovarian and endometrial cancer, and moderate levels have been found in lung cancer (12). In 2003, Hellstrom et al reported the potential role of HE4 as a secreted biomarker (22), after which time serum HE4 was investigated as a possible valuable biomarker for ovarian and endometrial cancers. In a meta-analysis, Zhen et al reported that the diagnostic accuracy of HE4 in distinguishing ovarian cancer from other benign gynecological diseases was found to be superior to that of CA125 (23). More recently, Brennan et al reported in a large population-based study that serum HE4 may be a useful prognostic biomarker in endometrial cancer (17). Furthermore, Yang et al determined its importance as a preoperative predictor for optimal tumour cytoreductive surgery in epithelial ovarian cancer (18). In 2012, researchers found that serum HE4 levels were significantly elevated in lung cancer patients compared with healthy controls (24). In 2014, Nagy et al, confirmed that HE4 levels were significantly elevated in lung cancer patients
Table II. Characteristics of colorectal cancer patients.

\begin{tabular}{lc}
\hline Variables & $\mathrm{n}(\%)$ \\
\hline HE4 & \\
Positive & $13(28.3)$ \\
Negative & $33(71.7)$ \\
Lymph node status & \\
Positive & $33(71.7)$ \\
Negative & $13(28.3)$ \\
CEA & \\
Positive & \\
Negative & $28(66.7)$ \\
CA199 & $14(33.3)$ \\
Positive & \\
Negative & $17(40.5)$ \\
Grade & $25(59.5)$ \\
1 & \\
2 & $13(35.1)$ \\
3 & $21(56.8)$ \\
Stage & $3(8.1)$ \\
I & \\
II & $22(4.3)$ \\
III & $11(23.9)$ \\
IV & $11(23.9)$ \\
\hline
\end{tabular}

${ }^{a} \mathrm{~A}$ CEA level 0-3.4 ng/ml was considered as negative and $>3.4 \mathrm{ng} / \mathrm{ml}$ as positive; and ${ }^{\mathrm{b} a} \mathrm{CA} 199$ level 0-37 U/ml was considered as negative and $>37 \mathrm{U} / \mathrm{ml}$ as positive according to our laboratory cut-off values. HE4, human epididymis protein 4; CEA, carcinoembryonic antigen; CA19-9, carbohydrate antigen 19-9.

compared with controls, and also demonstrated that HE4 levels were significantly correlated with tumour size and the presence of lymph node metastasis, similar to the findings of the present study (25). In another more recent study, again consistent with the present findings, Guo et al confirmed that HE4 was more commonly observed in gastric carcinoma tissues compared with normal tissues and was significantly correlated with advanced stage and tumour size (26); moreover, they found that 
Table III. Comparison of HE4 -positive and -negative patients.

\begin{tabular}{|c|c|c|c|}
\hline Variables & HE4-positive, n (\%) & HE4-negative, n (\%) & P-value \\
\hline Age, years $($ mean $\pm S D)$ & $63.3 \pm 11.7$ & $59.4 \pm 11.8$ & 0.315 \\
\hline Grade & & & 0.162 \\
\hline I & $2(16.7)$ & $11(44.0)$ & \\
\hline II & $8(66.6)$ & $13(52.0)$ & \\
\hline III & $2(16.7)$ & $1(4.0)$ & \\
\hline Lymph node status & & & 0.009 \\
\hline Positive & $13(100.0)$ & $20(60.6)$ & \\
\hline Negative & $0(0.0)$ & $13(39.4)$ & \\
\hline Stage & & & 0.009 \\
\hline I-II & $0(0.0)$ & $20(60.6)$ & \\
\hline III-IV & $13(39.4)$ & $13(100.0)$ & \\
\hline $\mathrm{CEA}, \mathrm{ng} / \mathrm{ml}$ & & & 0.159 \\
\hline$\geq 3.7$ & $11(84.6)$ & $17(58.6)$ & \\
\hline$<3.7$ & $2(15.4)$ & $12(41.4)$ & \\
\hline CA199, U/ml & & & $<0.001$ \\
\hline$\geq 37$ & $11(84.6)$ & $6(20.7)$ & \\
\hline$<37$ & $2(15.4)$ & $23(79.3)$ & \\
\hline
\end{tabular}

Bold print indicates statistical significance. HE4, human epididymis protein 4; CEA, carcinoembryonic antigen; CA19-9, carbohydrate antigen 19-9; SD, standard deviation.

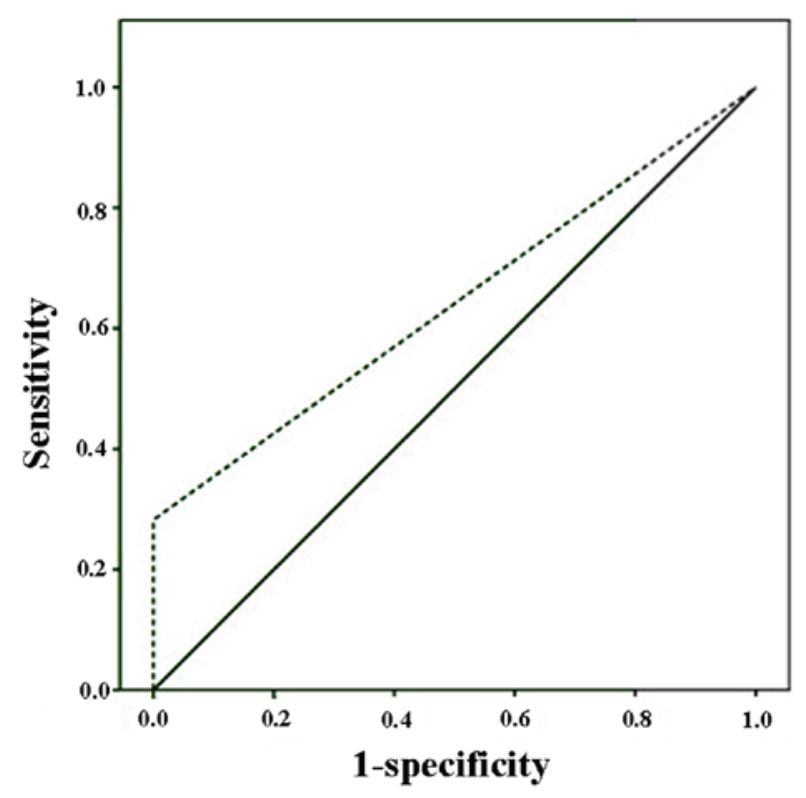

Figure 1. Receiver operating characteristic curve analyses of the diagnostic performance of human epididymis protein 4 in colorectal cancer patients and healthy controls.

silencing HE4 in vitro diminishes the phosphorylation level of Akt, Erk1/2, Fak and Src, all of which are important for the angiogenesis, migration and survival of cancer cells (26).

To the best of our knowledge, this is the first report to examine plasma HE4 levels in CRC patients. In the literature, only Galgano et al (12) have investigated HE4 expression in

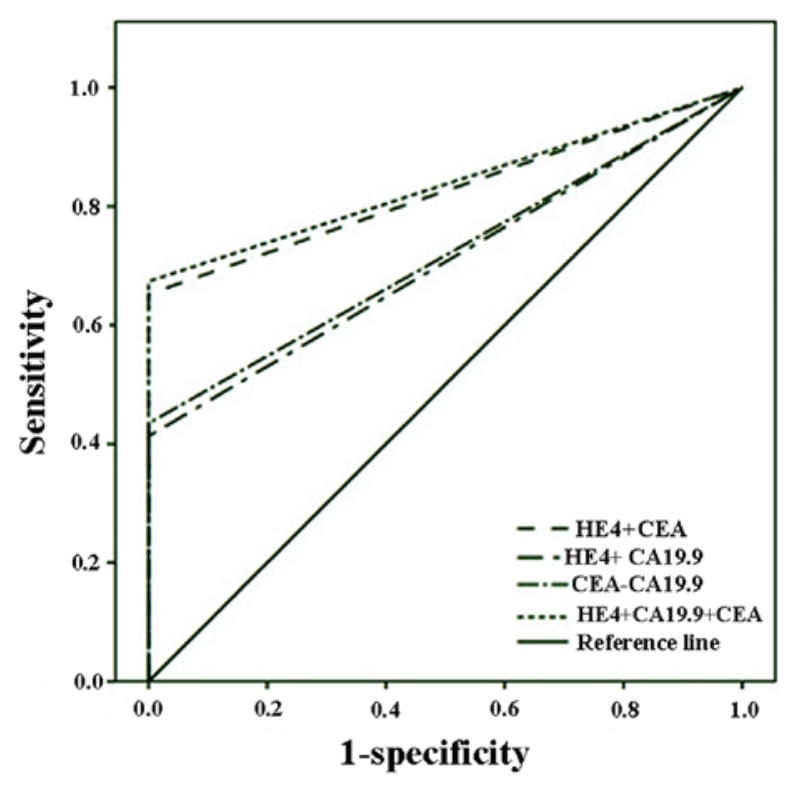

Figure 2. Receiver operating characteristic curve analyses of human epididymis protein 4 (HE4) combined with carcinoembryonic antigen (CEA) and carbohydrate antigen (CA) 19-9.

CRC tissues compared with other human neoplasms, and reported weak positivity in this group. The results of the present study not only show the significance of HE4 positivity in CRC, but also demonstrate the positive association with cancer stage and CA19-9 levels, which may be simply correlated with tumour load or be due to complex tumour biology. 
This study has certain limitations: First, the sample size was relatively small; and second, information on follow-up is lacking. However, it was designed as a preliminary study and has reached all the end points; thus, it may be considered a valuable basis for further research.

In conclusion, serum HE4 may be a useful biomarker in CRC, particularly in patients with stage III-IV disease. Further studies are required to confirm these findings and, in the future, HE4 may be used as a new target for therapeutic interventions.

\section{References}

1. Labianca R, Nordlinger B, Beretta GD, Mosconi S, Mandalà M, Cervantes A and Arnold D; ESMO Guidelines Working Group: Early colon cancer: ESMO clinical practice guidelines for diagnosis, treatment and follow-up. Ann Oncol 24 (Suppl 6): vi64-vi72, 2013

2. World Cancer Report 2014. World Health Organization. Chapter $1.1,2014$.

3. Welch JP and Donaldson GA: The clinical correlation of an autopsy study of recurrent colorectal cancer. Ann Surg 189: 496-502, 1979.

4. Borner MM: Neoadjuvant chemotherapy for unresectable liver metastases of colorectal cancer-too good to be true? Ann Oncol 10: 623-326, 1999.

5. Pamies RJ and Crawford DR: Tumor markers. An update. Med Clin North Am 80: 185-199,1996.

6. Perkins GL, Slater ED, Sanders GK and Prichard JG: Serum tumor markers. Am Fam Physician 68: 1075-1082, 2003.

7. Clinical practice guidelines for the use of tumor markers in breast and colorectal cancer. Adopted on May 17, 1996 by the American Society of Clinical Oncology. J Clin Oncol 14: 2843-2877, 1996.

8. Fletcher RH: Carcinoembryonic antigen. Ann Intern Med 104: 66-73, 1986.

9. Gupta MK, Arciaga R, Bocci L, Tubbs R, Bukowski R and Deodhar SD: Measurement of a monoclonal-antibody-defined antigen (CA19-9) in the sera of patients with malignant and nonmalignant diseases. Comparison with carcinoembryonic antigen. Cancer 56: 277-283, 1985.

10. Kirchhoff C, Habben I, Ivell R and Krull N: A major human epididymis-specific cDNA encodes a protein with sequence homology to extracellular proteinase inhibitors. Biol Reprod 45: 350-357, 1991.

11. Drapkin R, von Horsten HH,Lin Y, Mok SC, Crum CP, Welch WR and Hecht JL: Human epididymis protein 4 (HE4) is a secreted glycoprotein that is overexpressed by serous and endometrioid ovarian carcinomas. Cancer Res 65: 2162-2169, 2005.

12. Galgano MT, Hampton GM and Frierson HF Jr: Comprehensive analysis of HE4 expression in normal and malignant human tissues. Mod Pathol 19: 847-853, 2006.

13. Kirchhoff C: Molecular characterization of epididymal proteins. Rev Reprod 3: 86-95, 1998.
14. Clauss A, Lilja $\mathrm{H}$ and Lundwall A: A locus on human chromosome 20 contains several genes expressing protease inhibitor domains with homology to whey acidic protein. Biochem J 368: 233-242, 2002.

15. Thompson RC and Ohlsson K: Isolation, properties, and complete amino acid sequence of human secretory leukocyte protease inhibitor, a potent inhibitor of leukocyte elastase. Proc Natl Acad Sci USA 83: 6692-6696, 1986.

16. Plebani M; HE4 Study Group: HE4 in gynecological cancers: Report of a European investigators and experts meeting. Clin Chem Lab Med 50: 2127-2136, 2012.

17. Brennan DJ, Hackethal A, Metcalf AM, Coward J, Ferguson K, Oehler MK, Quinn MA, Janda M, Leung Y, Freemantle M, et al: Serum HE4 as a prognostic marker in endometrial cancer-a population based study. Gynecol Oncol 132: 159-165, 2014.

18. Yang Z, Luo Z, Zhao B, Zhang W, Zhang J, Li Z and Li L: Diagnosis and preoperative predictive value of serum HE4 concentrations for optimal debulking in epithelial ovarian cancer. Oncol Lett 6: 28-34, 2013.

19. Simmons AR, Baggerly K and Bast RC Jr: The emerging role of HE4 in the evaluation of epithelial ovarian and endometrial carcinomas. Oncology (Williston Park) 27: 548-556, 2013.

20. Yu S, Yang HJ, Xie SQ and Bao YX: Diagnostic value of HE4 for ovarian cancer: A meta-analysis. Clin Chem Lab Med 50: 1439-1446, 2012.

21. Lin J, Qin J and Sangvatanakul V: Human epididymis protein 4 for differential diagnosis between benign gynecologic disease and ovarian cancer: A systematic review and meta-analysis. Eur J Obstet Gynecol Reprod Biol 167: 81-85, 2013.

22. Hellstrom I, Raycraft J, Hayden-Ledbetter M, Ledbetter JA, Schummer M, McIntosh M, Drescher C, Urban N and Hellström KE: The HE4 (WFDC2) protein is a biomarker for ovarian carcinoma. Cancer Res 63: 3695-3700, 2003.

23. Zhen S, Bian LH, Chang LL and Gao X: Comparison of serum human epididymis protein 4 and carbohydrate antigen 125 as markers in ovarian cancer: A meta-analysis. Mol Clin Oncol 2: 559-566, 2014.

24. Iwahori K, Suzuki H, Kishi Y, Fujii Y, Uehara R, Okamoto N, Kobayashi M, Hirashima T, Kawase I and Naka T: Serum HE4 as a diagnostic and prognostic marker for lung cancer. Tumour Biol 33: 1141-1149, 2012.

25. Nagy B Jr, Bhattoa HP, Steiber Z, Csobán M, Szilasi M, Méhes G, Müller M, Lázár J, Kappelmayer J and Antal-Szalmás P: Serum human epididymis protein 4 (HE4) as a tumor marker in men with lung cancer. Clin Chem Lab Med 52: 1639-1648, 2014.

26. Guo YD, Wang JH, Lu H, Li XN, Song WW and Zhang XD: The human epididymis protein 4 acts as a prognostic factor and promotes progression of gastric cancer. Tumour Biol 36: 2457-2464, 2015. 